\title{
The Central Stars of the Planetary Nebulae NGC 7027 and NGC 6543
}

\author{
P.A.M. van Hoof ${ }^{1}$, D.A. Beintema ${ }^{2}$, F. Lahuis ${ }^{3}$ and S.R. Pottasch ${ }^{1}$ \\ ${ }^{1}$ Kapteyn Astronomical Institute, Groningen; \\ ${ }^{2}$ SRON Laboratory for Space Research, Groningen; \\ ${ }^{3}$ Agencia Espacial Europea, Villafranca del Castillo, Madrid
}

Infrared spectra of NGC 7027 and NGC 6543 ranging from $2.4 \mu \mathrm{m}$ to $45 \mu \mathrm{m}$ were obtained with the Short Wavelength Spectrometer on board the Infrared Space Observatory. A first analysis of these spectra, with the aid of photo-ionization models, is presented in Beintema et al. (1996).

We report the first detection of the [Ar VI] $4.53 \mu \mathrm{m}$ and [Ne VI] $7.65 \mu \mathrm{m}$ lines in the spectrum of NGC 7027 (Fig. 1). When compared with older observations it is clear that the [Ar VI] line and possibly also other lines have increased in strength since 1981 . We argue that a likely explanation for this variability is a change in the spectral energy distribution of the central star, possibly an increase in effective temperature. However, this result needs to be confirmed by further observations.

We also report a non-detection of the [O IV] $25.9 \mu \mathrm{m}$ line and the first detection of the [Na III] $7.32 \mu \mathrm{m}$ line in the spectrum of NGC 6543. The non-detection is not expected based on a blackbody approximation for the spectrum of the central star. The ionization threshold for $\mathrm{O}^{3+}$ is just beyond the He II limit, and the absence of this line shows that the stellar flux drops at least by a factor 350 at the He II limit. Modeling the [O IV] line may prove to be a valuable test for atmosphere models.
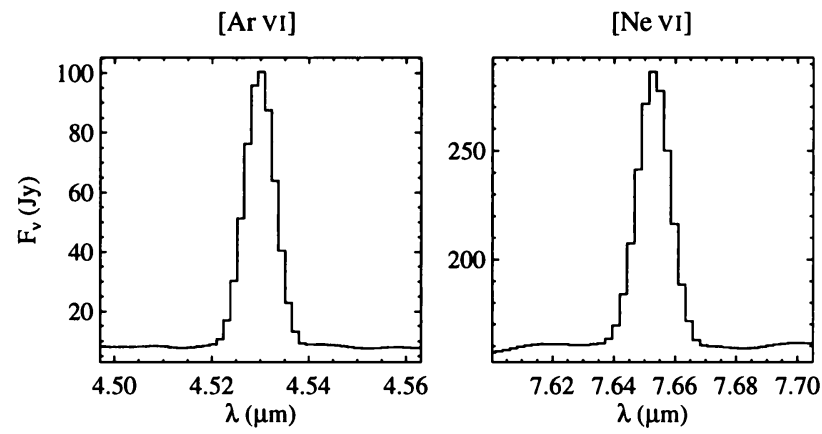

Figure 1: The observed [Ar VI] and [Ne VI] lines in the spectrum of NGC 7027.

\section{REFERENCES}

Beintema D.A., van Hoof P.A.M., Lahuis F. et al., 1996, A\&A, 315, L253 